\title{
Towards passive station holding of autonomous underwater vehicles inspired by fish behaviour in unsteady flows
}

\author{
AB Phillips ${ }^{1}$, JIR Blake $^{1}$, SW Boyd ${ }^{1}$ and G Griffiths ${ }^{2}$ \\ ${ }^{1}$ Faculty of Engineering and the Environment, University of Southampton, UK \\ ${ }^{2}$ National Oceanography Centre, Southampton, UK \\ Email: abp@ soton.ac.uk
}

\begin{abstract}
Some species of fish are able to alter their mode of swimming to interact with naturally produced vortices; the use of these gaits reduces the energy expended by the fish. To analyse the feasibility of autonomous underwater vehicles (AUV) replicating these gaits, a series of experiments are performed with unpowered rigid and flexible bodies positioned in the Kármán wake of a rigid cylinder. Simple motion capture techniques are used to capture the bodies' lateral and upstream motion in the flow. The results demonstrate that manufactured bodies are capable of passively mimicking fish behaviours, to a limited extent. More importantly, it was concluded that while significant upstream movement was possible for a manufactured object, it was achievable irrespective of the stiffness of the material. For AUVs operating in unsteady flow regimes an ability to utilise energy saving gaits may improve the range or operational time.
\end{abstract}

\section{Introduction}

Uneven or rocky sea or river beds, sea-ice structures, man-made structures, waves and coastal surf zones coupled with a local current or flow lead to unsteady non-uniform flows. Evolution has equipped most fish with a lateral line, a sensory system comprising of organs known as mechanoreceptive neuromasts which allow fish to sense these flow features and take advantage of unsteady flows to minimise energy expenditure. The preference of fish to use unsteady flow features has been observed in both the laboratory ${ }^{1,2}$ and the field ${ }^{3-5}$. Improved understanding of the behaviour of fish in unsteady flows is important for stream design, habitat management and fish passageway design ${ }^{6}$. 
The flow past a rigid cylinder held perpendicular to a uniform flow is a useful example of a time varying flow, which has been extensively studied ${ }^{7}$. A characteristic wake, termed a Kármán vortex street, is observed comprising of two staggered rows of vortices shed from alternative sides of the cylinder, with an alternating direction of rotation. These vortices slowly dissipate due to viscosity as they travel downstream. Directly behind the cylinder there exists a region where the wake exhibits a net upstream average velocity, this region is termed the 'suction' region and typically extends up to two cylinder diameters downstream. Two distinct methods for fish to interact with these flow features have been observed. Entraining is where the fish maintains its position in earth fixed co-ordinates directly behind the body with their head just behind and to one side of the cylinder ${ }^{1}$. The effect of the low-pressure suction region behind the bluff body means the fish makes no regular axial swimming motions and yet can still balance thrust and drag forces to hold station behind the bluff body. Slaloming is where the fish will seek and maintain a position a few body lengths downstream of a bluff body, outside of the suction region, where it seeks to 'slalom' between shed vortices in a technique that is termed the Kármán gait ${ }^{8}$, see Figure 1 . The fish voluntarily modify their body kinematics to interact with the vortices present in the flow, the fish synchronize their tailbeat frequency to the vortex shedding frequency of the cylinder and display large lateral movements. Such behaviour has been observed in fish with a variety of body shapes and occupying different ecological niches. Results in the literature show that both mechanisms result in station holding relative to the earth frame of reference, thus suggesting that the hydrodynamic thrust and drag forces are equal and opposite ${ }^{9}$. 

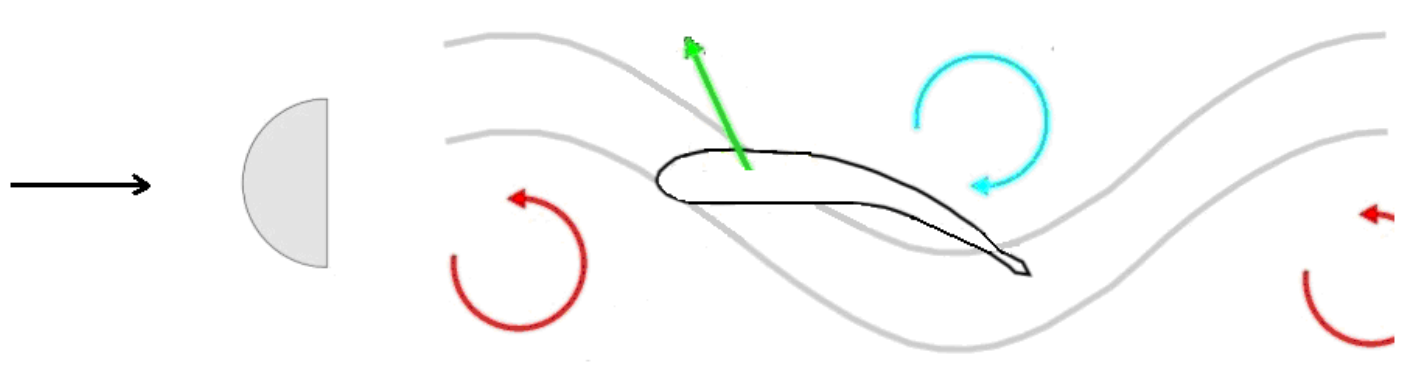

B

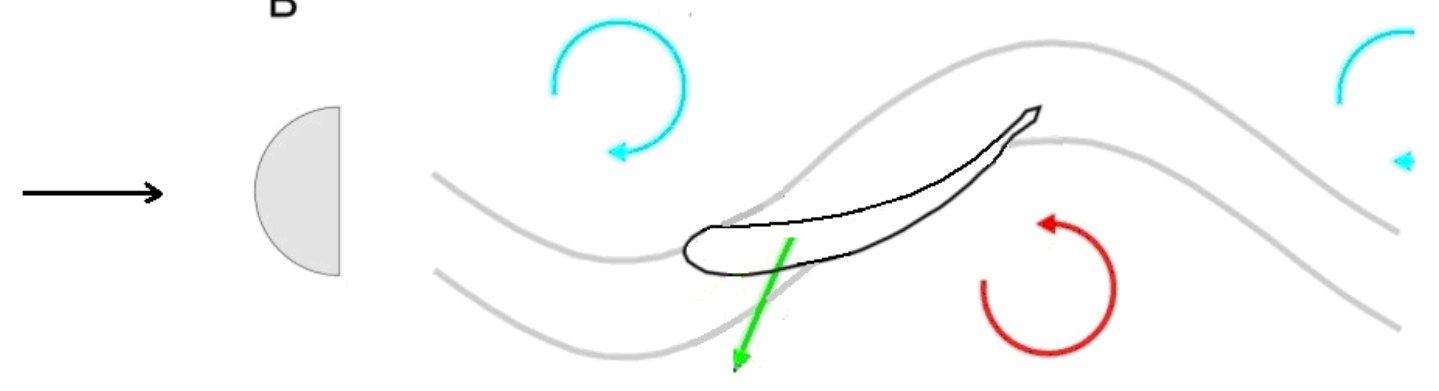

Figure 1. Schematic of the hydrodynamic mechanism of the Kármán gait (adapted from Liao et al. ${ }^{8}$ ). The fish 'slaloms' between shed vortices. The resulting thrust and drag acting on the fish balance resulting in no net upstream or downstream motion.

Based on theoretical and experimental work with oscillating foils, live and euthanized fish, Liao et al..$^{8,9}$ hypothesized that the mechanism of vortex capture for thrust production in a vortex street can be accounted for as a result of the body interacting with the dynamics in the wake. Liao et al ${ }^{8}$ performed flow visualization around a rainbow trout (Oncorhynchus mykiss) swimming in the unsteady wake of a D-section cylinder. They were able to show that the trout adopted the Kármán gait in preference to other modes of swimming. On monitoring the muscle activity of trout swimming behind a bluff body, when adopting the Kármán gait, the only muscles used were the anterior axial ones; an activity mainly associated with station keeping and not propulsion. This is in contrast to normal swimming (in a free stream) where a pattern of propagating muscle activity down the length of the fish was observed. Compared to trout swimming in the free stream at 4.5 $\mathrm{BL} \mathrm{s}^{-1}$ (Body Lengths per second), trout holding station behind the large cylinder at the same flow speed showed a significant decrease (303\%) in tailbeat frequency and a large (354\%) increase in wavelength passing down the body. This matches the body oscillations to the vortex shedding frequency and spacing. In addition, body amplitudes (lateral movement of the body) increase $300-800 \%$, depending on body location. Rainbow trout are lotic (stream- 
dwelling) fish, thus they are expected to have evolved a body shape and behaviours to take advantage of the obstacles present in these habitats.

This observation is confirmed by the work of Beal et al ${ }^{10}$, where a dead trout tied to a string and placed in the wake of a bluff body was also observed to adopt a Kármán gait like motion and travel upstream. Later studies by Liao et al. ${ }^{9}$ showed that the dead trout only exhibited signs of moving upstream prior to rigor mortis, after which no upstream movement was observable. As a consequence, it was suggested that the natural resonance of a still-flexible dead trout is crucial in determining its ability to move upstream, since it is unable to actively modify its body kinematics. In an experiment to determine if these ideas could be applied to engineered systems

Phillips et al. $^{11}$ demonstrated the ability of a slender flexible body to adopt the Kármán gait and exhibit repeatable upstream motion. Based on these observations it is hypothesised that a rigid body would be unable to adopt the Kármán gait and travel upstream. Toming et al. ${ }^{12}$ measured the hydrodynamic forces acting on fish like bodies held at fixed downstream locations in a Kármán vortex street, they demonstrated that the forces induced on the body would cause the fish to sway and yaw.

It is clear from the experiments with inanimate objects that an active process that modifies body kinematics is not required in order to achieve the Kármán gait. However, to maintain position for significant durations some form of active process is required in order to maintain orientation with respect to the shed vortices and to prevent excessive upstream or lateral motions. These motions can result in the object being drawn further into the suction zone were the objects exhibit entraining or the object interacting with the free stream velocity and being convected downstream. This work aims to examine ways of developing a bio-inspired Autonomous Underwater Vehicle (AUV) around these considerations; the potential gains of which are clearly evident when the speed and manoeuvrability of conventional AUVs are compared to their marine animal equivalents. To further our understanding on the behaviour of neutrally flexible and rigid bodies operating in unsteady flows, video capture techniques are used to study the oscillations of anguilliform shaped bodies located in the von Kármán wake. In particular we test the hypothesis that a rigid body would be unable to adopt the Kármán gait.

\section{Experimental Method}

Experiments were performed at the University of Southampton Armfield Water Channel at the Chilworth Science Park; characteristics of which are listed in Table 1. Variable flow speeds are achieved using a 
combination of three pumps. Partial filling of the tank constrained the flow rate to a smaller area, thus yielding a larger flow velocity.

Table 1. Tank characteristics for the Southampton University Armfield water channel.

\begin{tabular}{cccc}
\hline \multicolumn{4}{c}{ Tank Characteristics (Armfield Water Channel): } \\
\hline Length $[L]=$ & 23.0 & $\mathrm{~m}$ & \\
Width $[W]=$ & 1.5 & $\mathrm{~m}$ & \\
Depth $[D]=$ & 0.6 & $\mathrm{~m}$ & $(\max )$ \\
Flow rate $[Q]=$ & 0.8 & $\mathrm{~m}^{3} / \mathrm{s}$ & $(\max )$ \\
Kinematic viscosity $[v]=$ & $1.156 \times 10^{-6}$ & $\mathrm{~m}^{2} / \mathrm{s}$ & $\left(\right.$ at $\left.15^{\circ} \mathrm{C}\right)$
\end{tabular}

Figure 2 illustrates the experimental setup. An unsteady Kármán wake was generated by holding a rigid circular cylinder of $0.09 \mathrm{~m}$ diameter $\left(D_{b}\right)$ perpendicular (vertical) to a flow with an unconstrained velocity of $U_{0}$. The top of the cylinder was fixed rigidly to an existing arrangement that rests over the top of the water channel. The bottom of the cylinder was fitted to a concentric disc securing the cylinder between the bottom of the water channel and the top fixture. The disc had a diameter 1.25 times larger than that of the cylinder to which it is fixed. Hence, the local flow velocity at the bottom of the cylinder is increased, creating a bottom end condition which is conducive to parallel vortex shedding. Such measures are in accordance to those taken by Khalak \& Williamson ${ }^{13}$. Downstream of the cylinder the free surface was covered with a Perspex sheet to minimise disturbance of the free surface and to minimise distortions of the video image.

The inflow flow speed was varied from 0.36 to $0.5 \mathrm{~m} / \mathrm{s}$, resulting in a Reynolds number $\left(\operatorname{Re}=U_{0} D_{b} / v\right)$ range of between 32000 and 45000 . The $6 \%$ constriction due to the presence of the cylinder increases the effective flow at the cylinder, $U_{c}$ :

$$
U_{c}=U_{0}\left(\frac{W}{W-D_{b}}\right)
$$

The frequency at which vortices are shed from a bluff body is characterized by the Strouhal number:

$$
S t_{c}=\frac{f_{c} D_{b}}{U_{c}}
$$

where $f_{c}$ is the frequency of vortex formation, $W$ is the width of the channel and $U_{c}$ is the constricted flow velocity. The number indicates how often vortices are shed from the bluff body. The Strouhal number is a 
function of Reynolds number but remains relatively constant at a value of approximately 0.2 for a wide range of Reynolds numbers $\left(10^{2}\right.$ to $\left.10^{5}\right)$. Based on particle image velocimetry PIV observations, Liao et al. ${ }^{2}$ suggest that the lateral vortex spacing may be taken as the cylinder diameter and that the downstream vortex spacing (distance between shed vortices of the same sign) may be calculated from:

$$
\lambda=\frac{U_{0}}{f_{c}}
$$

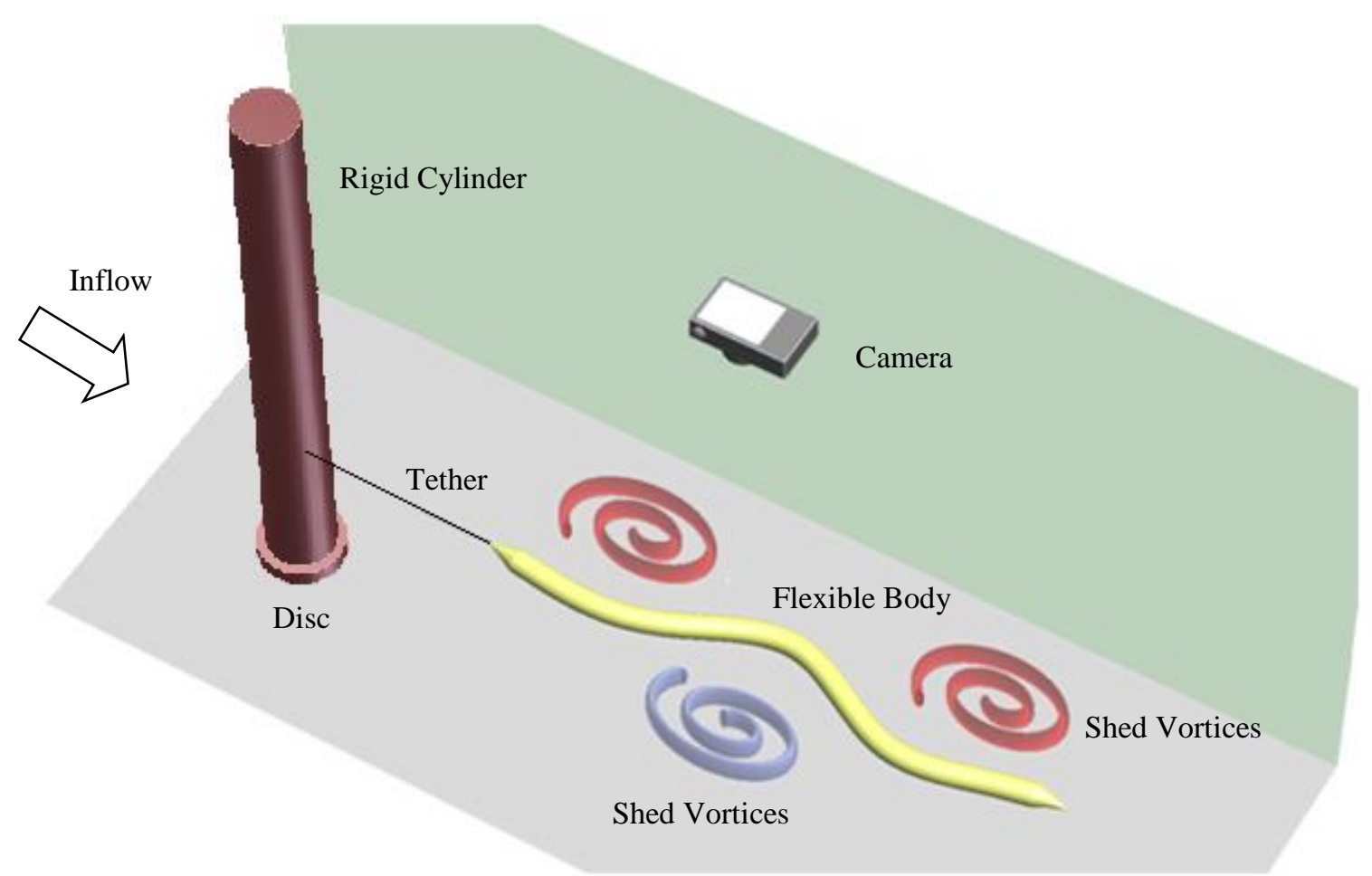

Figure 2. Experimental setup outlining the resulting fluid-structure interactions to a given flow configuration.

The flexible body detailed in Table 2 was manufactured by feeding a silicone molding compound (Moldsil ${ }^{\mathrm{TM}}$ ) into a cylindrical mold. An air bubble was trapped down the center of the mold to achieve neutral buoyancy. 
Table 2. Physical characteristics of flexible body.

\begin{tabular}{ccc}
\hline \multicolumn{3}{c}{ Flexible body characteristics: } \\
\hline Length $\left[L_{c}\right]=$ & 0.675 & $\mathrm{~m}$ \\
Parrallel midbody & 0.597 & $\mathrm{~m}$ \\
Diameter $\left[D_{c}\right]=$ & 0.026 & $\mathrm{~m}$ \\
Young's Modulus $\left[E_{c}\right]=$ & 0.319 & $\mathrm{MPa}$ \\
Mass per unit length $[\mu]=$ & $5.428 \times 10^{-1}$ & $\mathrm{~kg} / \mathrm{m}$ \\
\hline
\end{tabular}

Two types of tether were used to attach the flexible body to the rigid cylinder. The first tether (cat 4 Ethernet cable) prevents upstream and downstream motion but allows limited lateral motion at the nose and is referred to as the 'flexible tether'. The second tether is tension only $(0.25 \mathrm{~mm}$ diameter steel wire $)$ which allows the downstream cylinder to 'swim' upstream or travel laterally, but not travel downstream further than the tether's length and referred to as a 'tension only tether'.

\section{Natural Frequency Analysis}

In order to understand the behavior of the flexible body it is useful to understand the mode shapes and natural frequencies of the body. For the flexible body these were estimated by performing a simple modal analysis (Block Lanczos method) using ANSYSтм Mechanical v12. A 20-node structural solid element (SOLID95) was used to discretise the geometry, using a global mesh size of $0.003 \mathrm{~m}$ at full scale.

Solution of the finite element equations requires that sufficient boundary conditions are applied to the cylinder. The upstream nose of the cylinder is fixed in all degrees of freedom, while the downstream end is free to move. This does not accurately replicate the boundary conditions acting on the tethered flexible body but provides indicative results for the structural response of the cylinder, see figure 3. 


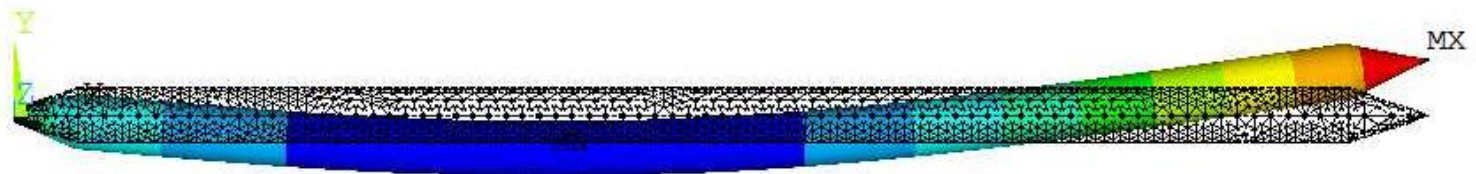

Figure 3. The zeroth (top) and first (bottom) mode shapes of the fixed-free flexible body.

The associated natural frequencies are predicted to be $0.06 \mathrm{~Hz}$ and $0.7 \mathrm{~Hz}$, in a vacuum. These frequencies will be lower for the flexible body surrounded by a fluid of density, $\rho_{w}$, due to the inertia of the surrounding fluid increasing the effective mass of the flexible body. An initial estimate of the submerged frequencies may be made by assuming the added mass of the cylinder is equivalent to $\frac{\rho_{w} \pi D_{c}{ }^{2}}{4}$ per unit length and modifying the density of the material in ANSYS ${ }^{\mathrm{TM}}$ appropriately. This approach suggests that the zeroth and first natural frequencies are $0.03 \mathrm{~Hz}$ and $0.5 \mathrm{~Hz}$ when submerged in water.

\section{Motion Tracking Analysis}

For each run the pumps were turned on and allowed to settle at a constant speed. The motion of the flexible body was then captured for five minutes. Each case was repeated at least once. A video camera (Canon IXUS 95 running at 30fps with a $640 \times 480$ resolution) was used to track the movement of the flexible body from above. In an effort to identify the distortion modes as exhibited by the flexible body, a MATLAB script was developed to run a sequence of image data processing operations on the recorded frames. To minimize the likelihood of extracting superfluous image data and to increase the contrast, a number of dark bands were marked on the body at equidistant lengths along the parallel midbody.

The MATLAB script's primary function is to compare each pixel intensity value to a threshold intensity value and subsequently allowing a binary image to be generated of all the pixels that meet the imposed user criteria, see figure 4. In this image all the pixels corresponding to dark markings are shown in white. The centroid of each of the markings is then identified. These centroids have been superimposed on the final image in figure 4 . 

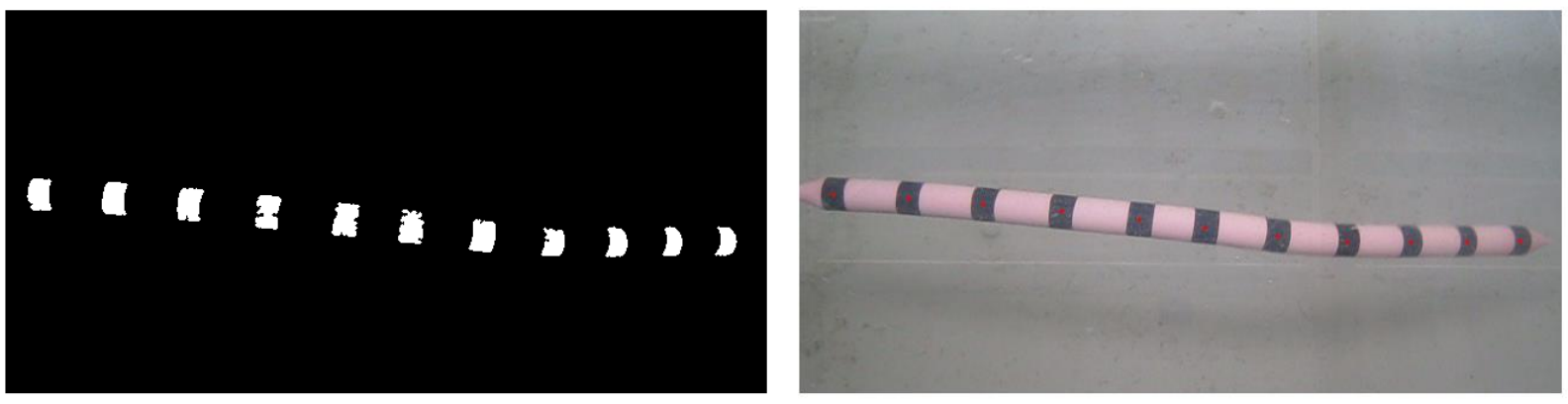

Figure 4. Threshold intensity markings redisplayed in B\&W (left) manipulated to determining their respective centroid locations. Centroid locations superimposed on the colour image (right).

Once all the appropriate results were extracted, validation on each centroid's location had to be performed prior to carrying out any further analysis. Occasionally the MATLAB script failed to extract the appropriate centroid position. Typically, this was due to the presence of bubbles in the flow. This error was rectified manually and since this operation was extremely time consuming it was decided to resample the video data at $6 \mathrm{~Hz}$ rather than $30 \mathrm{~Hz}$ for these manual manipulations. This is well above the Nyquist sampling rate, the minimum sampling rate in order to avoid aliasing, given our shedding frequencies of interest lie between 0.86 and $1.2 \mathrm{~Hz}$, see Table 3 .

Given that the flexible object is expected to lie in a plane parallel to the field of view of the camera, which is also parallel to the bottom of the tank, one can assume that by knowing both the length in pixels between the nose and tail in addition to the full scale length between such markings, a factor scaling one to the other can be determined. Knowing this scale factor, it is then possible to determine the displacement in time of each marking. Observations of the bodies' motion in the vertical plane showed limited vertical bending and pitching of the flexible body. The pitch angle did not exceed 10 degrees and therefore due to the cosine term the resulting errors in the position where less than $2 \%$.

\section{Results}

A series of different speeds and downstream locations were investigated. However, only 6 cases are presented: -

Case 1 - Flexible body, flexible tether, $2 D_{b}$ downstream, $U_{0}=0.36 \mathrm{~m} / \mathrm{s}$

Case 2 - Flexible body, flexible tether, $2 D_{b}$ downstream, $U_{0}=0.5 \mathrm{~m} / \mathrm{s}$

Case 3 - Flexible body, flexible tether, $3 D_{b}$ downstream, $U_{0}=0.36 \mathrm{~m} / \mathrm{s}$

Case 4 - Flexible body, flexible tether, $3 D_{b}$ downstream, $U_{0}=0.5 \mathrm{~m} / \mathrm{s}$

Case 5 - Flexible body, tension only tether, $3 D_{b}$ downstream, $U_{0}=0.42 \mathrm{~m} / \mathrm{s}$ 
Case 6 - Rigid body, tension only tether, $3 D_{b}$ downstream, $U_{0}=0.42 \mathrm{~m} / \mathrm{s}$

Table 3 summarises the flow conditions for the three water channel speeds. Experiments were performed with the nose of the cylinder initially positioned two diameters downstream, usually estimated as the downstream extent of the suction zone and at three diameters downstream.

Table 3. First four applied fluid flow cases and their respective outcomes.

\begin{tabular}{|c|c|c|c|c|}
\hline Nominal flow velocity $[U]=$ & 0.362 & 0.498 & 0.417 & $\overline{\mathrm{m} / \mathrm{s}}$ \\
\hline Constricted flow velocity $\left[U_{c}\right]=$ & 0.385 & 0.53 & 0.444 & $\mathrm{~m} / \mathrm{s}$ \\
\hline Water depth $=$ & 0.41 & 0.41 & 0.405 & $\mathrm{~m}$ \\
\hline Bluff body Reynolds no. $\left[R e_{b}\right]=$ & $3.00 \times 10^{4}$ & $4.13 \times 10^{4}$ & $3.45 \times 10^{4}$ & \\
\hline Strouhal no. $\left[S t_{c}\right]=$ & 0.190 & 0.188 & 0.190 & \\
\hline Flexible body Reynolds no. $\left[R e_{m}\right]=$ & $1.99 \times 10^{5}$ & $2.74 \times 10^{5}$ & $2.30 \times 10^{5}$ & \\
\hline Vortex shedding frequency $\left[f_{c}\right]=$ & 0.856 & 1.18 & 0.987 & $\mathrm{~Hz}$ \\
\hline Predicted downstream vortices spacing $[\lambda]=$ & 0.423 & 0.423 & 0.423 & $\mathrm{~m}$ \\
\hline
\end{tabular}

Analysis of the video footage suggests that the vortex shedding frequency is within $4 \%$ of theoretical. In all cases a small amount of hogging (mid-length of the flexible body floating higher than the ends) was observed in the flexible body due to differences in the weight and buoyancy distribution.

\subsection{Case 1 - Flexible body, flexible tether, $2 D_{b}$ downstream, $U_{0}=0.36 \mathrm{~m} / \mathrm{s}$}

As the shed vortices pass by the flexible body the resulting pressure field and shear forces acting on the flexible body induce lateral motion (figure 5). The magnitude of the oscillations increases from the nose to the tail. A phase lag is apparent in the results between the nose and the tail, due to the time it takes the vortex to move downstream. The lateral motion at the tail seldom exceeds 1.2 times the radius of the rigid cylinder. The stiffness of the tether prevents significant upstream motion, and limits the extent of lateral motion at the nose. 


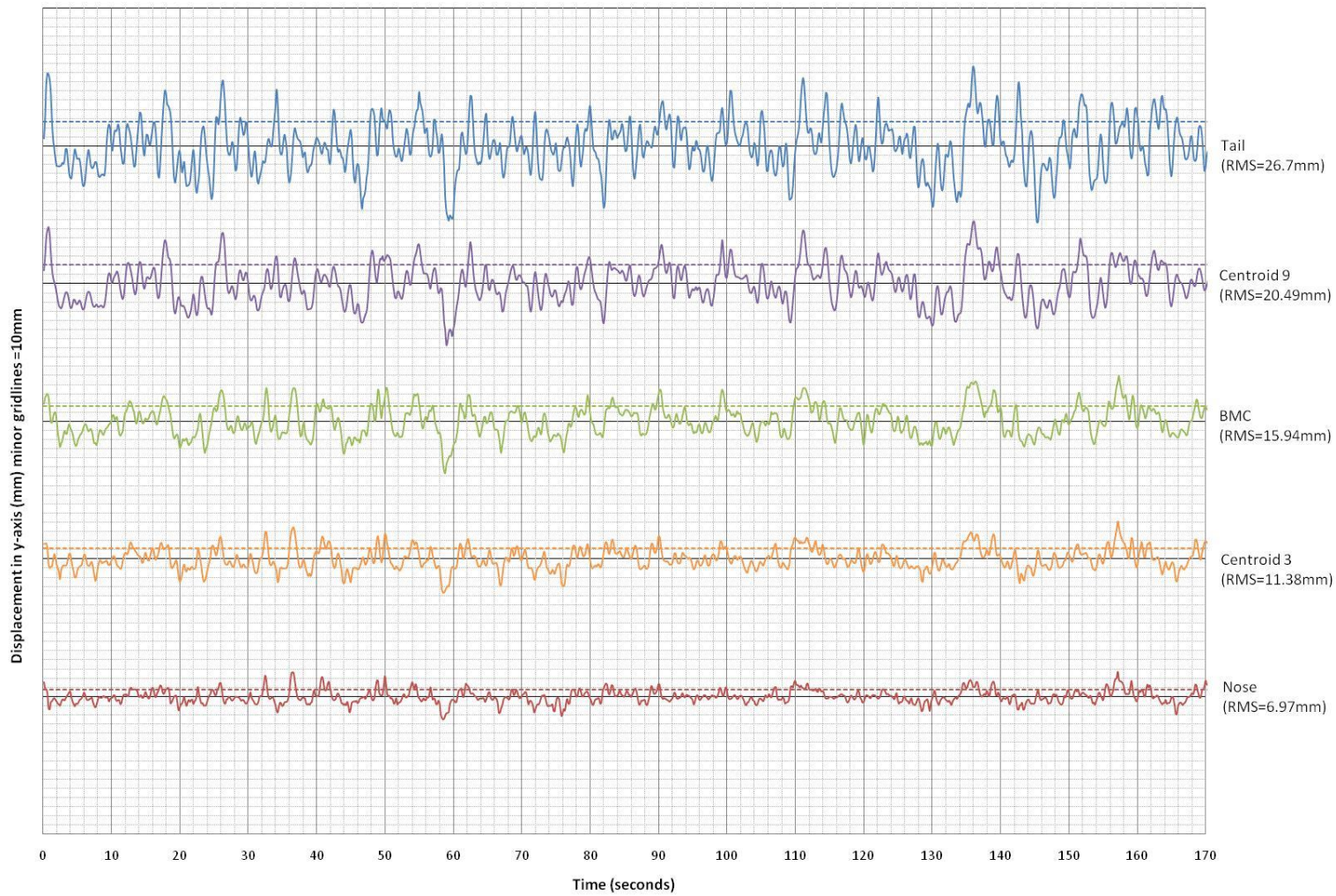

Figure 5. Lateral excursions for flexible body, flexible tether, of length $2 D_{b} U_{0}=0.36 \mathrm{~m} / \mathrm{s}, f_{c}=0.856 \mathrm{~Hz}$. The dashed lines correspond to the root mean square (RMS) of the lateral motion.

To help visualise the motion described in Figure 5, Figure 6 illustrates a separate time series with dye in the flow. By looking at the first eight shots (frames 599 to 634 - spaced equally by five frames) it is easily observed that the flexible body does tend to flex in accordance to the respective vortex passing along its length, in what appears to be synchronised slaloming. The observed vortex spacing is comparable to the predicted $0.423 \mathrm{~m}$. This results in three vortices being present along the length of the flexible body at any time. The core of each vortex (highlighted by the red dye) has an associated region of low pressure due to the high local velocities. From the video footage it does appear that the flexible body is pulled laterally into the regions of low pressure, generating a travelling waveform along the flexible body. The resulting body deformations are similar to those of anguilliform swimmers, ${ }^{14}$. The observed motions suggest that the flexible body is producing some forwards thrust, hence would attempt to 'swim' upstream if the tether allowed, it's upstream progress would be determined by the balance of the thrust a drag forces acting on the body. Since the inflow to the rigid cylinder is turbulent the resulting downstream flow is not truly periodic: the strength and downstream spacing of the vortices have some variation, which may account for the flexible body not adopting a regular periodic motion. 

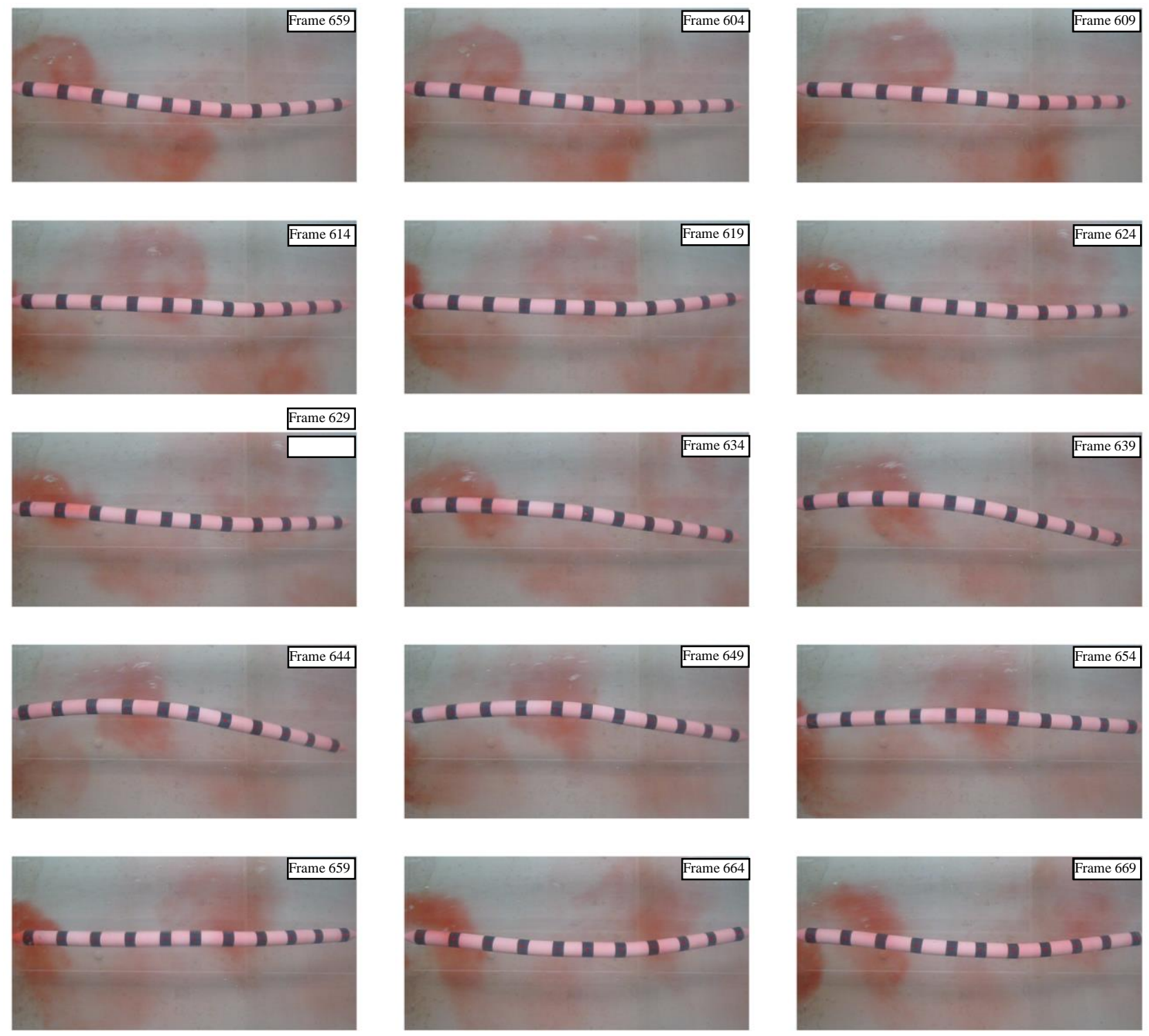

Figure 6. Frames 599 to 669 in 5 frame $(0.1667 \mathrm{~s})$ interval separation, $U_{0}=0.36 \mathrm{~m} / \mathrm{s}, f_{c}=0.856 \mathrm{~Hz}$. The flow is passing from left to right.

Using a fast Fourier transform (FFT) a power spectrum illustrating the frequency content of the flexible body's motions may be extracted. Figure 7 illustrates such a power spectrum at three locations along the cylinder: at the nose, mid length and tail. Both the nose and tail exhibit motions over a range of frequencies between 0.8 and $1.0 \mathrm{~Hz}$, corresponding closely to the calculated vortex shedding frequency of $0.856 \mathrm{~Hz}$. This region corresponds closely to both the predicted and expected vortex shedding frequency for this case, which confirms the visual observation that the flexible body is responding to the shed vortices. The FFT analysis also includes peaks corresponding to lower frequencies, noticeably at $0.08 \mathrm{~Hz}$ and $0.5 \mathrm{~Hz}$ : these values are in the region of the 
predicted frequencies of the zeroth and first natural frequencies of the flexible body from the finite element analysis. Frames 644 to 699 of Figure 6 do appear to show the body adopting the predicted first mode shape.. 


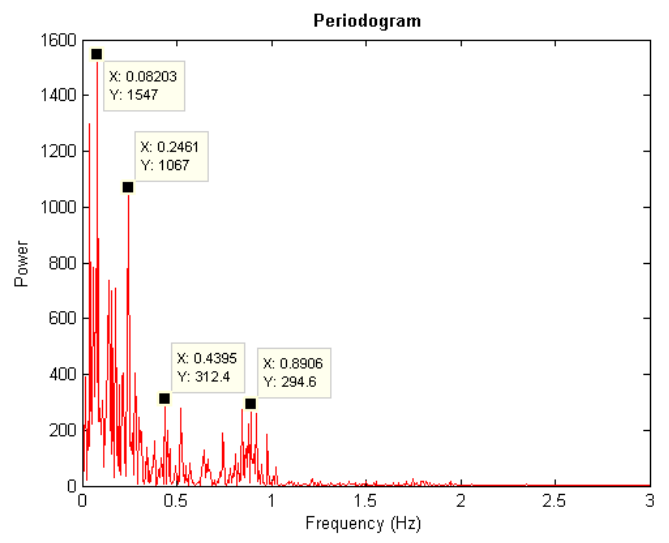

a) Case 1 - Nose

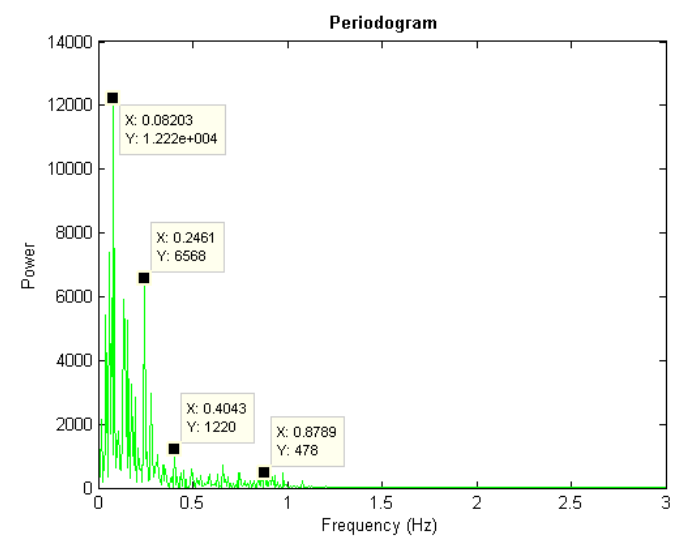

c) Case 1- Midbody

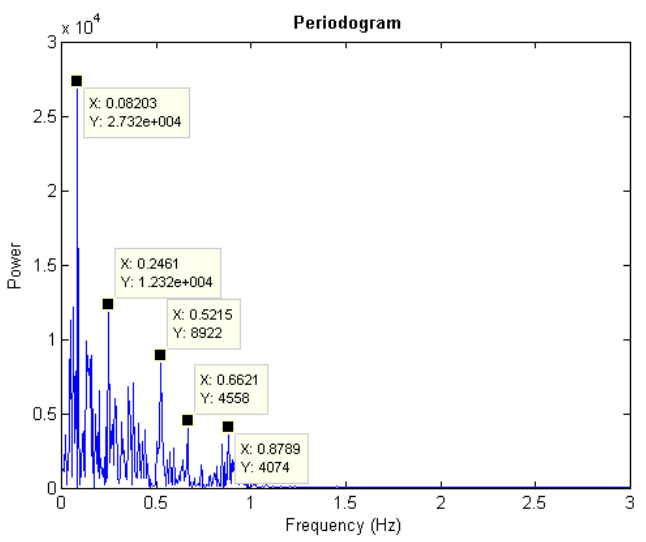

e) Case 1 - Tail

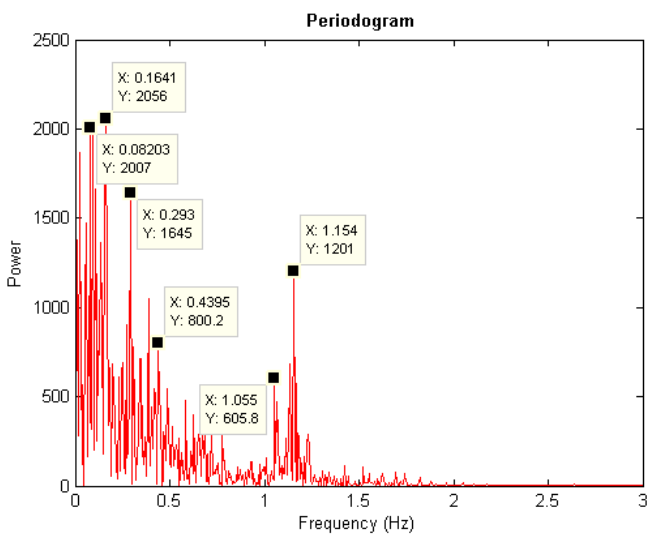

b) Case 2 - Nose

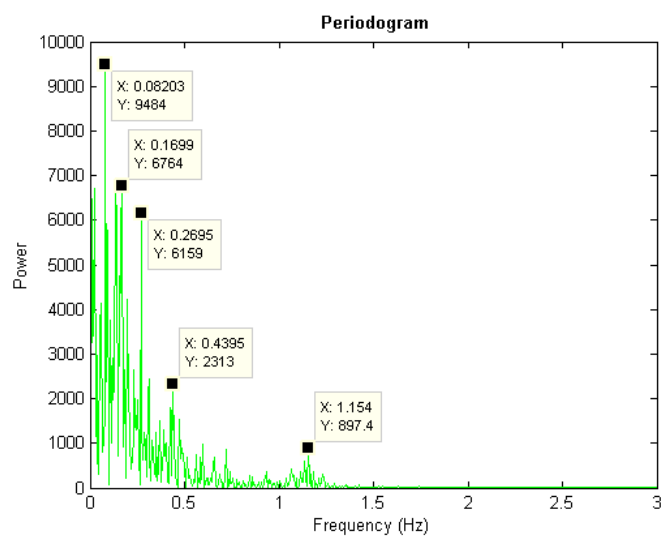

d) Case 2 - Midbody

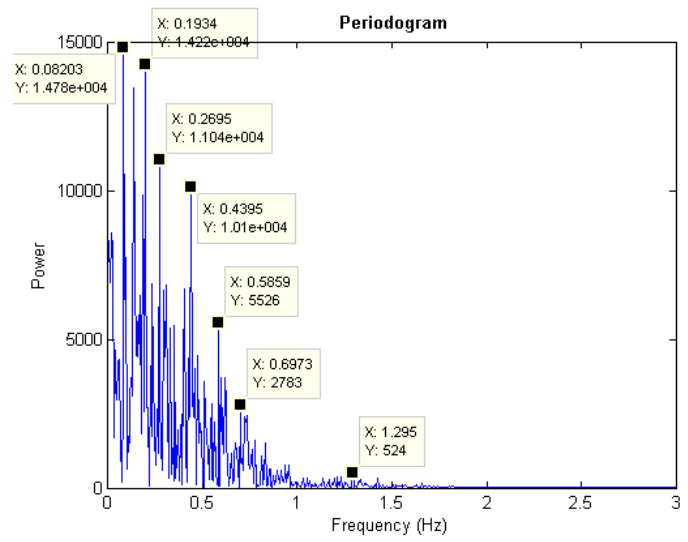

f) Case 2 - Tail

Figure 7. Fast Fourier Transform analysis of lateral motion, at nose (top left), BMC (top right) and Tail (bottom), for Case $1 f_{c}=0.856 \mathrm{~Hz}$ and Case $2 f_{c}=1.18 \mathrm{~Hz}$. 


\subsection{Case 2 - Flexible body, flexible tether, $2 D_{b}$ downstream, $U_{0}=0.5 \mathrm{~m} / \mathrm{s}$}

Increasing the flow velocity increases the vortex shedding frequency; this is illustrated in the time histories for this case (see figure 8). This change is observed in the frequency power spectrum, where the highest frequency peak is shifted to approximately $1.15 \mathrm{~Hz}$, which is close to the predicted vortex shedding frequency of $1.18 \mathrm{~Hz}$, see figure 7. The increased flow speed increases the number of trapped bubbles in the flow. These are clearly observed in Figure 9.

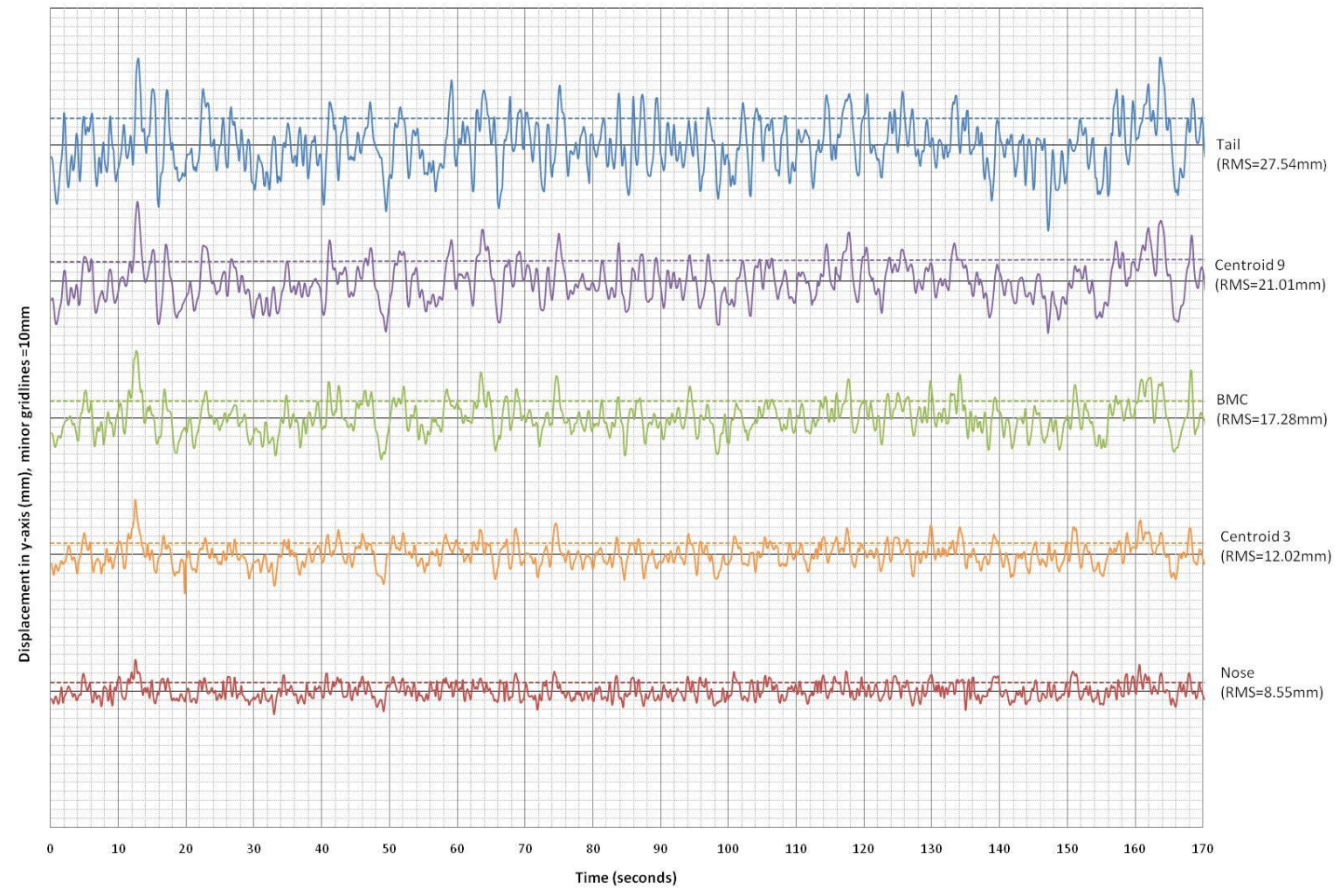

Figure 8. Lateral excursions for flexible body, flexible tether, of length $2 D_{b} U_{0}=0.5 \mathrm{~m} / \mathrm{s}, f_{c}=0.8144 \mathrm{~Hz}$. 

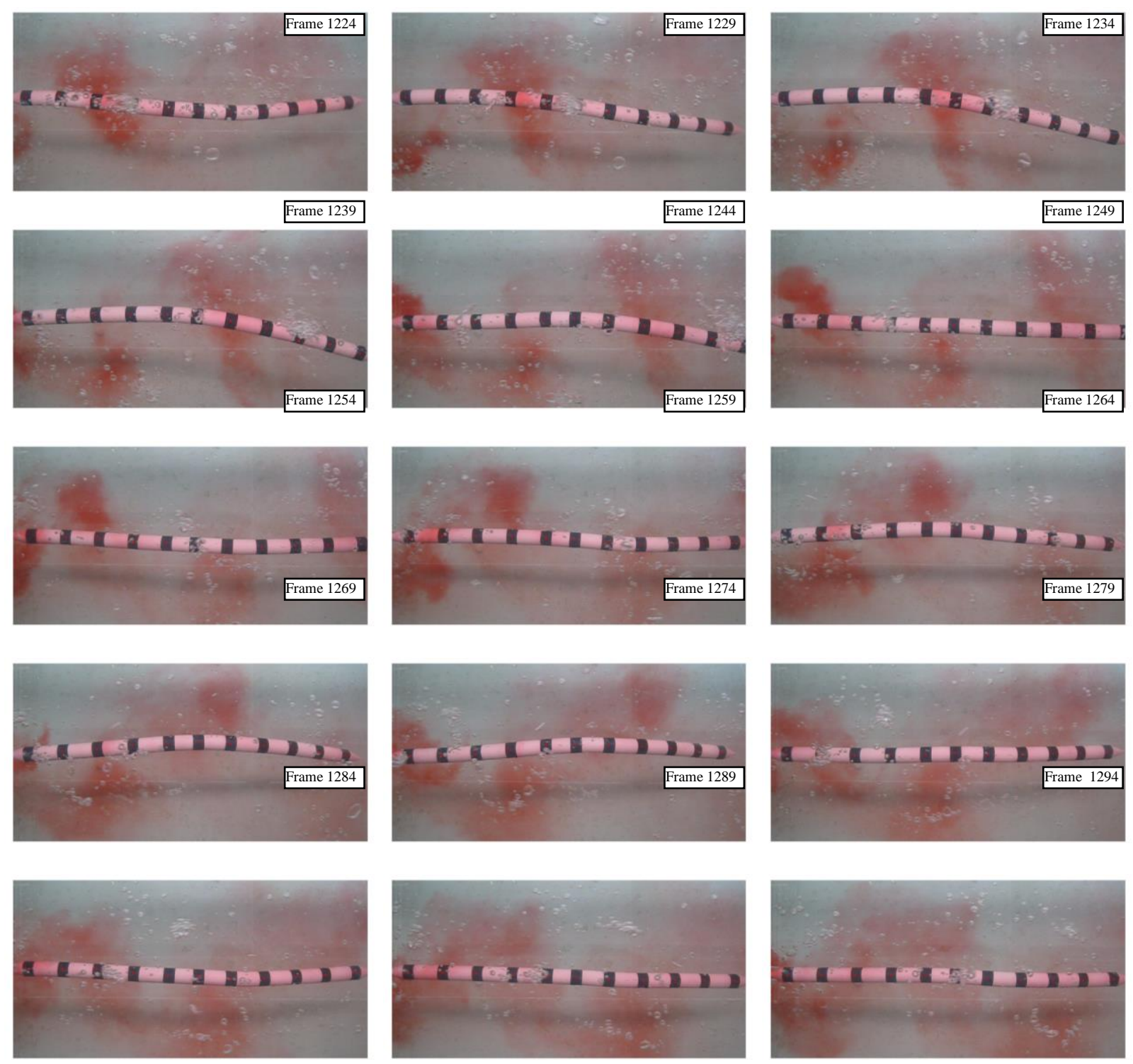

Figure 9. Case 2: Frames 1224 to 1294 in 5 frame $(0.1667 \mathrm{~s})$ intervals, $f_{c}=0.8144 \mathrm{~Hz}$. The flow is passing from left to right.

\subsection{Case $3 \& 4-$ Flexible body, flexible tether, $3 D_{b}$ downstream, $U_{0}=0.36 \mathrm{~m} / \mathrm{s}$ and $U_{0}=0.5 \mathrm{~m} / \mathrm{s}$}

In an attempt to further understand the role that the downstream position and thus the formation length of the mean recirculation region plays on the object's performance, the flexible body was placed at an alternate downstream location three times the diameter of the bluff body $\left(3 D_{b}\right)$. For these cases the body exhibited greater lateral motion at all positions along the body, see figure 10. The increased lateral motion may be partly attributed to the reduction in the lateral stiffness of the tether with increasing length. Thus the same forcing would result in increased lateral motion. The figure does show the greatest increase in lateral motion at the nose. 
The increase may also be attributed to the growth of the Kármán wake with downstream distance.

Similar to the results with both live and dead rainbow trout ${ }^{10}$ the tail amplitude is more than twice that observed at the nose. The increase in amplitude from nose to tail is approximately linear apart from a small kink at around $0.8 \mathrm{~L}$. This could potentially be attributed to the marking at $\mathrm{X} / \mathrm{L}=0.8$ being positioned too far forward, and hence resulting in a reduced RMS value. However, observation of the video footage suggests that the tail region of the flexible body is not truly horizontal due to the difference in the weight and buoyancy distributions along the body, which result in the flexible body hogging (nose and tail lower than midbody). This may also lead to inaccuracies in the lateral position measurement at these locations.

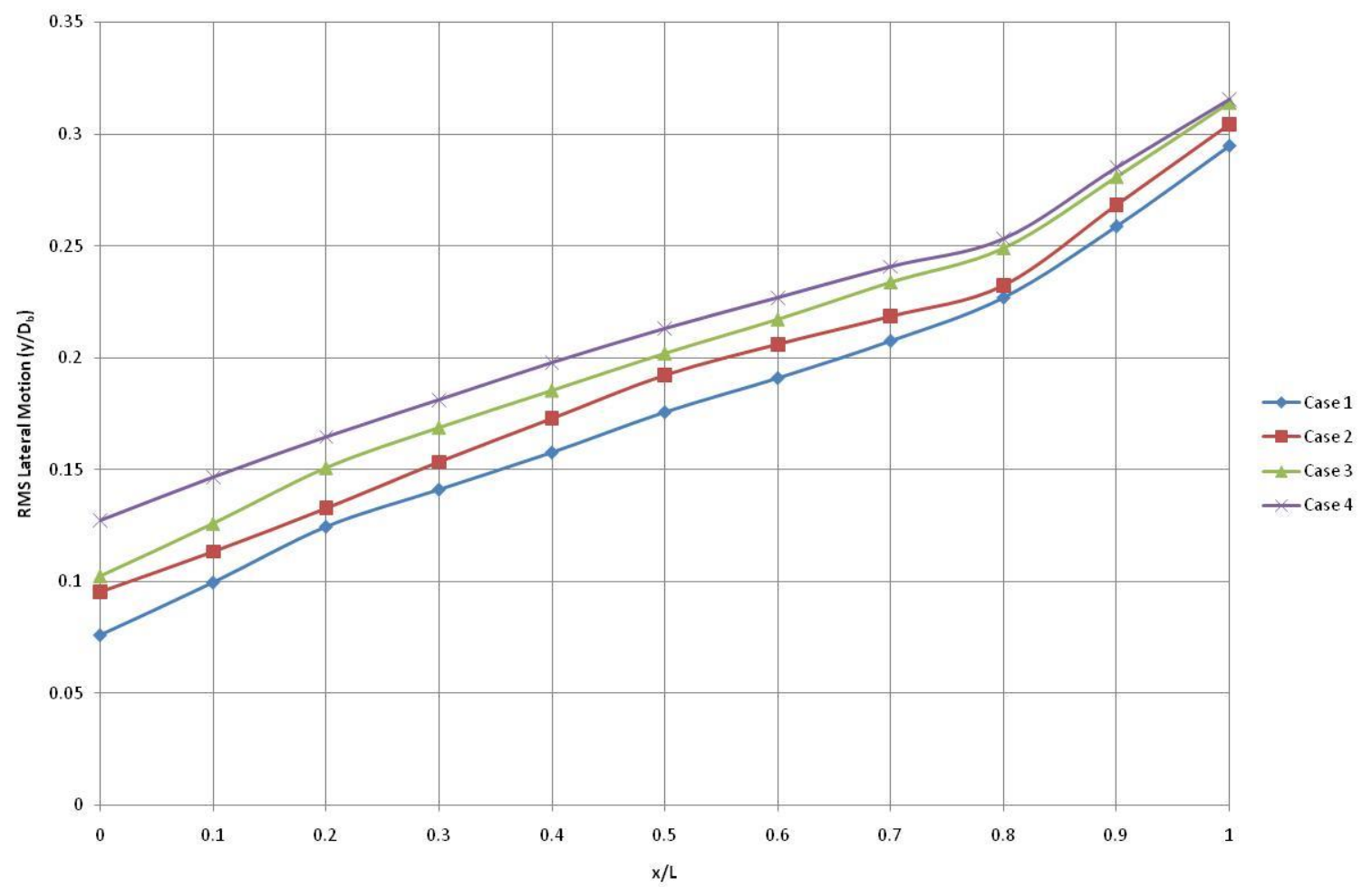

Figure 10. Root mean square of lateral motion for the first four cases considered.

\subsection{Case 5 - Flexible body, tension only tether, $3 D_{b}$ downstream, $U_{0}=0.42 \mathrm{~m} / \mathrm{s}$}

The previous four cases clearly show the flexible body interacting with the shed vortices from the rigid cylinder. However, the boundary conditions applied to the flexible body prevent large upstream excursions at the nose of the cylinder. For this case the tether is replaced with $0.25 \mathrm{~mm}$ steel cable. The uniform inflow is set to $0.417 \mathrm{~m} / \mathrm{s}$ which provides a good balance between high inflow velocity and low number of trapped bubbles. The shed vortices have a frequency of approximately $0.99 \mathrm{~Hz}$. 


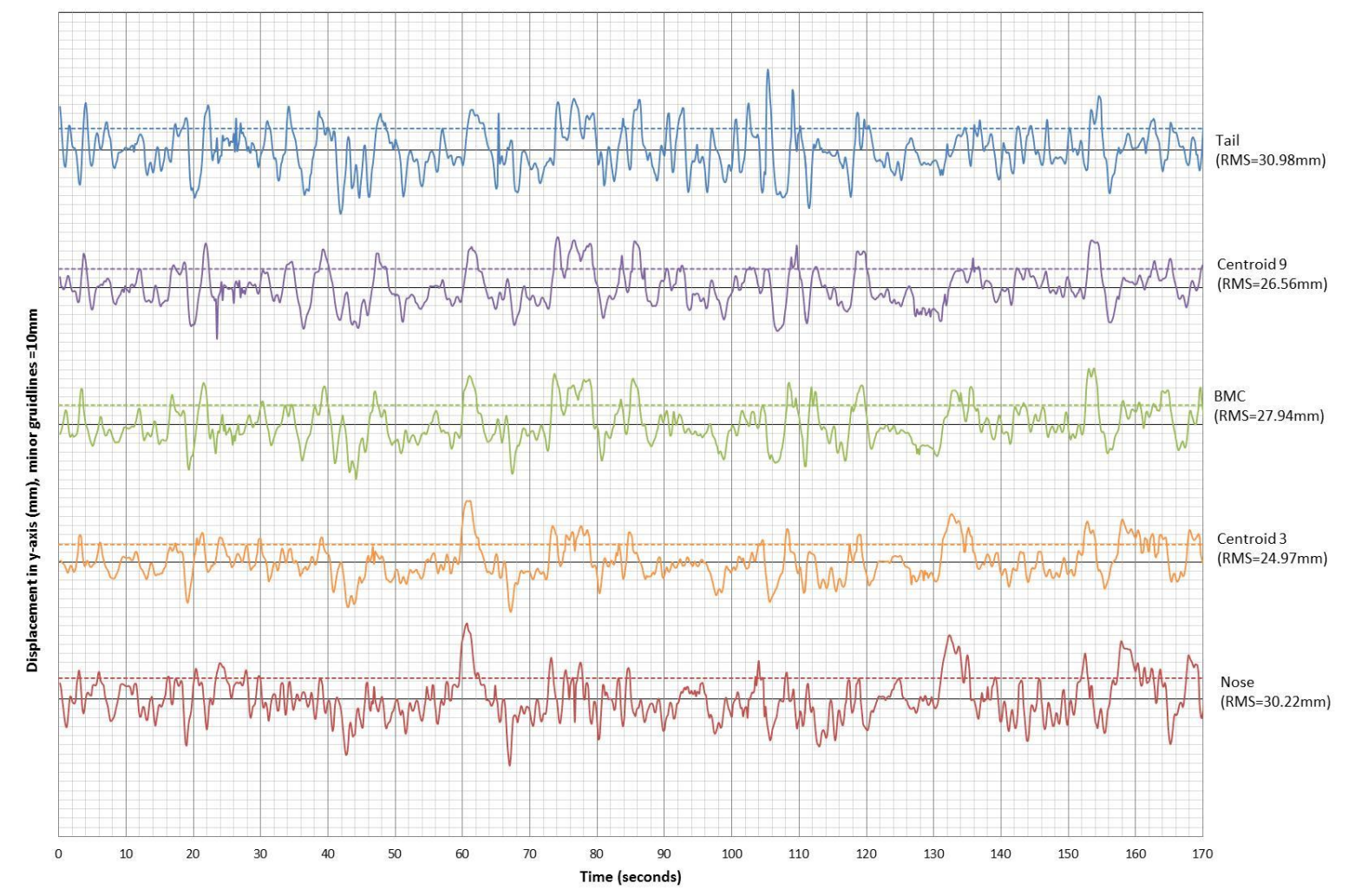

Figure 11. Lateral excursions for flexible body, tension only tether, of length $3 D_{b}, U_{0}=0.42 \mathrm{~m} / \mathrm{s}, f_{c}=0.93 \mathrm{~Hz}$.

Upon running the test, it was immediately obvious that the new tether had significantly changed the dynamics of the body: lateral oscillation of the nose was larger and upstream movement was common and of a considerable magnitude and duration. Starting from 3 diameters downstream of the rigid cylinder the flexible body would readily interact with the shed vortices; travelling waves were clearly identifiable down the length of the body, for example see time $=60.5 \mathrm{~s}$ on Figure 11 , a large amplitude event initiates at the nose and then propagates down the body occurring at the nose approximately 1s later. In contrast to the previous cases the amplitude of the lateral motion is much more constant along the length of the flexible body. This motion leads to upstream 'swimming' in a manner similar to the Kármán gait. Once the flexible body reached the 'suction region' it would be rapidly pulled into the low pressure region behind the cylinder. The nose would then move round to the separation point on one side or other of the cylinder, analogous to the entraining behaviour observed in fish, see Figure 12. Typically the nose would then stay in this location being forced forwards by thrust generated by 
the body, and then forced back as the nose moved past the separation point into the main flow. Similar behaviour was observed with dead rainbow trout by Beal et al. ${ }^{10}$. Live rainbow trout were also observed advancing towards the cylinder during the initial phase of Liao et al. ${ }^{2}$ experiments, where they were rapidly drawn upstream. Once the fish became accustomed to the flow they were then able to maintain station, by adopting the Kármán gait, for long periods of time.

Occasionally at some point in the cycle some disturbance would influence the motion of the flexible body and it would rapidly return to a downstream location. It was observed that these events correspond to part of the flexible body exiting the wake region and interacting with the surrounding free stream flow.

Unlike the live rainbow trout observed by Liao et al. ${ }^{2}$, the flexible body adopting the Kármán gait does not hold station using this motion, it is typically a transient phase from a distance downstream to a position upstream where the nose of the flexible body is positioned close to the flow separation point on one side of the cylinder. Entrainment appears to then become the primary station holding mechanism. This suggests that entraining may have a lower energetic requirement since the flexible body was able to hold station very close to the cylinder with no external power. These observations agree with the hypothesis that the small anterior muscle activity of the rainbow trout are associated with maintaining station in the flow using the Kármán gait. 

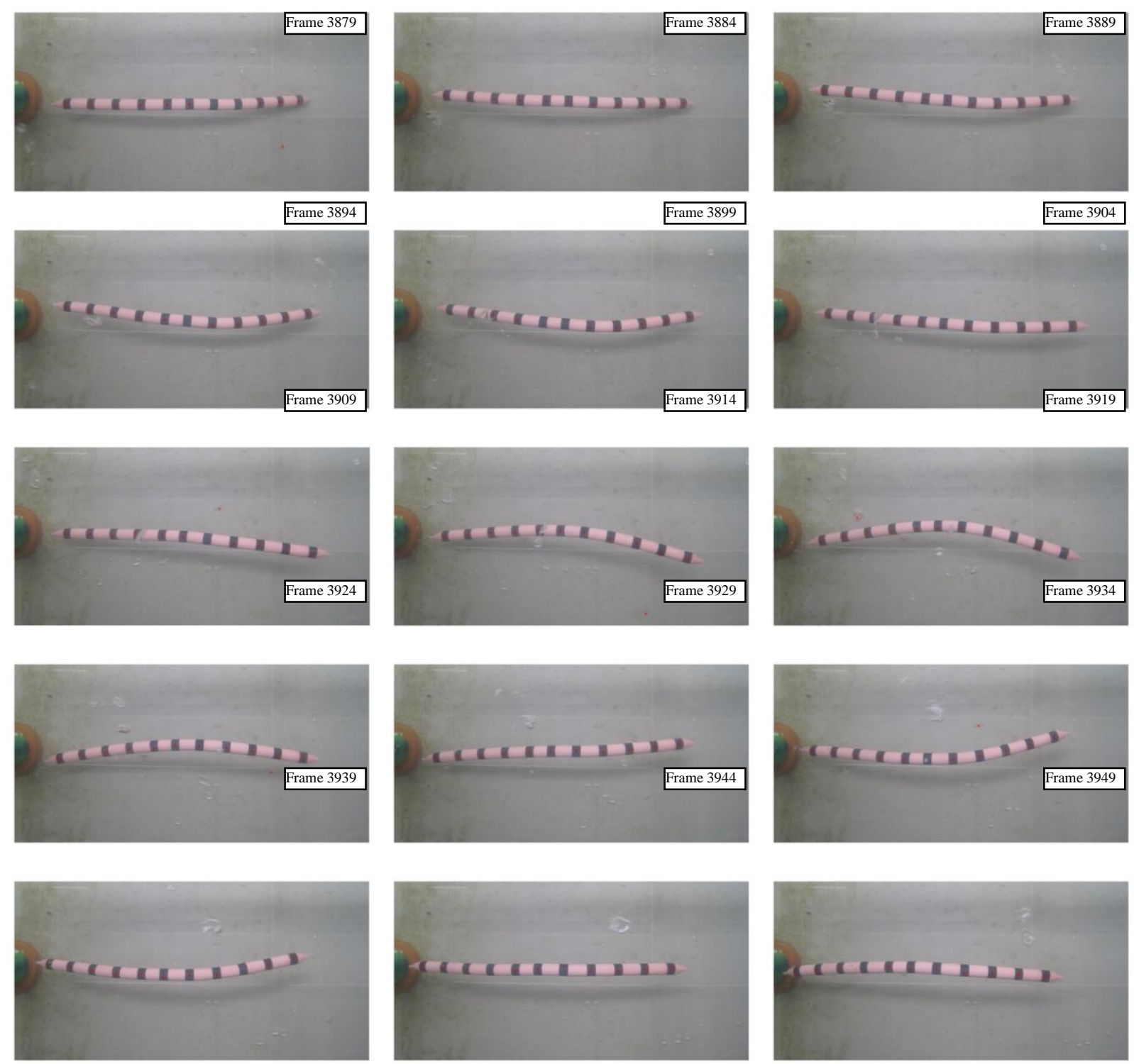

Figure 12. Upstream swimming of the flexible body, frames 3879 to 3949 , in 5 frame $(0.1667 \mathrm{~s})$ intervals. The flow is passing from left to right. Flow from left to right.

Figure 13 illustrates the upstream motion of the flexible body. Noting its downstream location with the nose positioned at $X=3 D_{b}$ the flexible body spends a significant proportion of its time upstream of this position. Due to the tether the nose cannot be downstream of this location. On three occasions the nose of the flexible body advances to the downstream extent of the rigid cylinder, $X=O D_{b}$. Due to lateral and vertical excursions of the nose the tether may come into tension at locations slightly upstream of $X=3 D_{b}$. Such occurrences are obvious on the figure. 


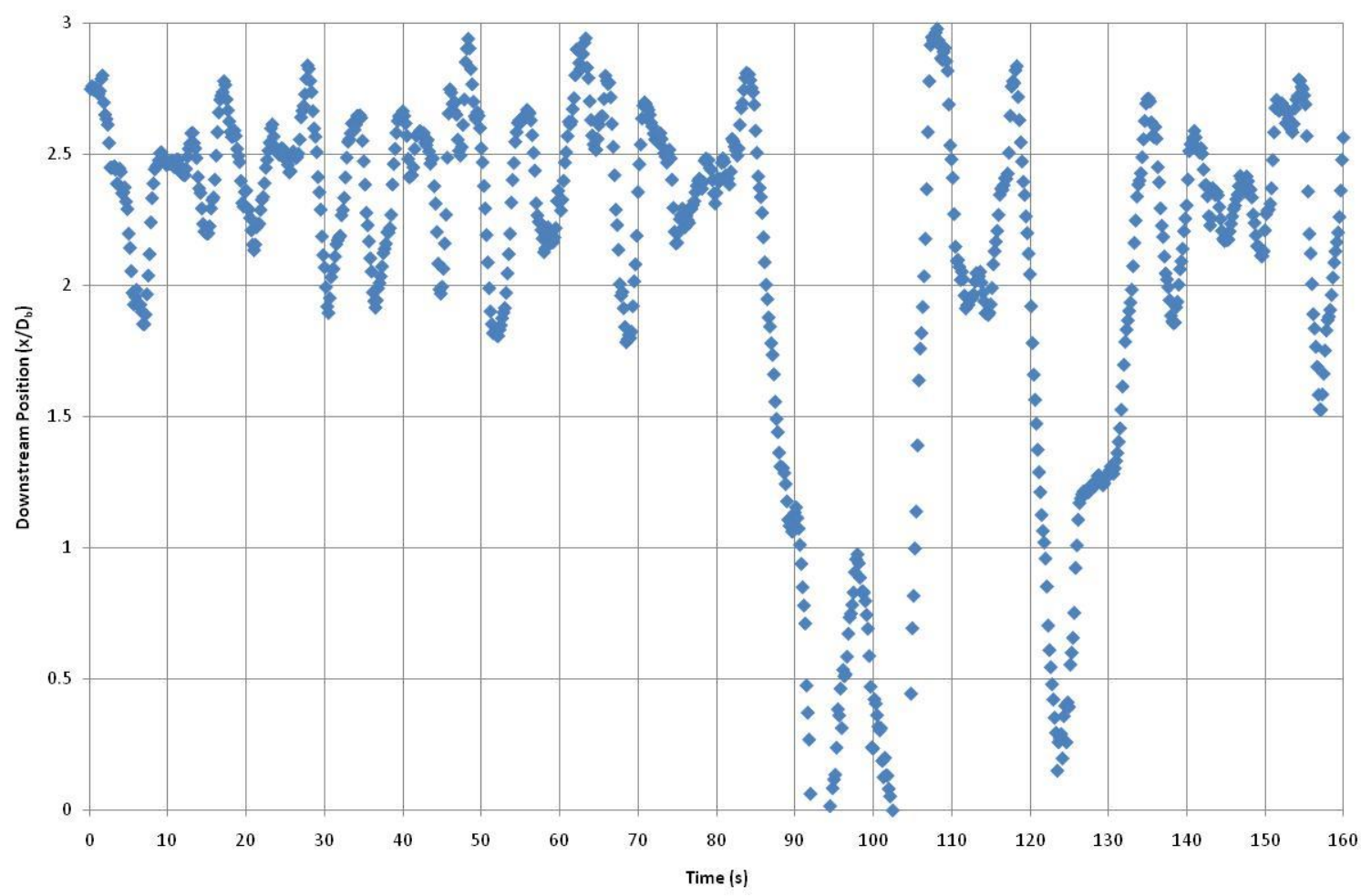

Figure 13. Upstream motion of the nose of the flexible body.

Figure 14 illustrates cumulative frequency for two three-minute data series. Both show good agreement for the time spent more than $2.5 D_{b}$ downstream. However, notable differences occur in the distribution of time spent upstream of this location. In one time history the flexible body was caught in the suction region on two occasions and 'pulled' rapidly upstream, this did not occur in the other sample. The interaction of the flexible body with the suction region can have a significant effect on the proportion of time the flexible body spends upstream of its initial position. 


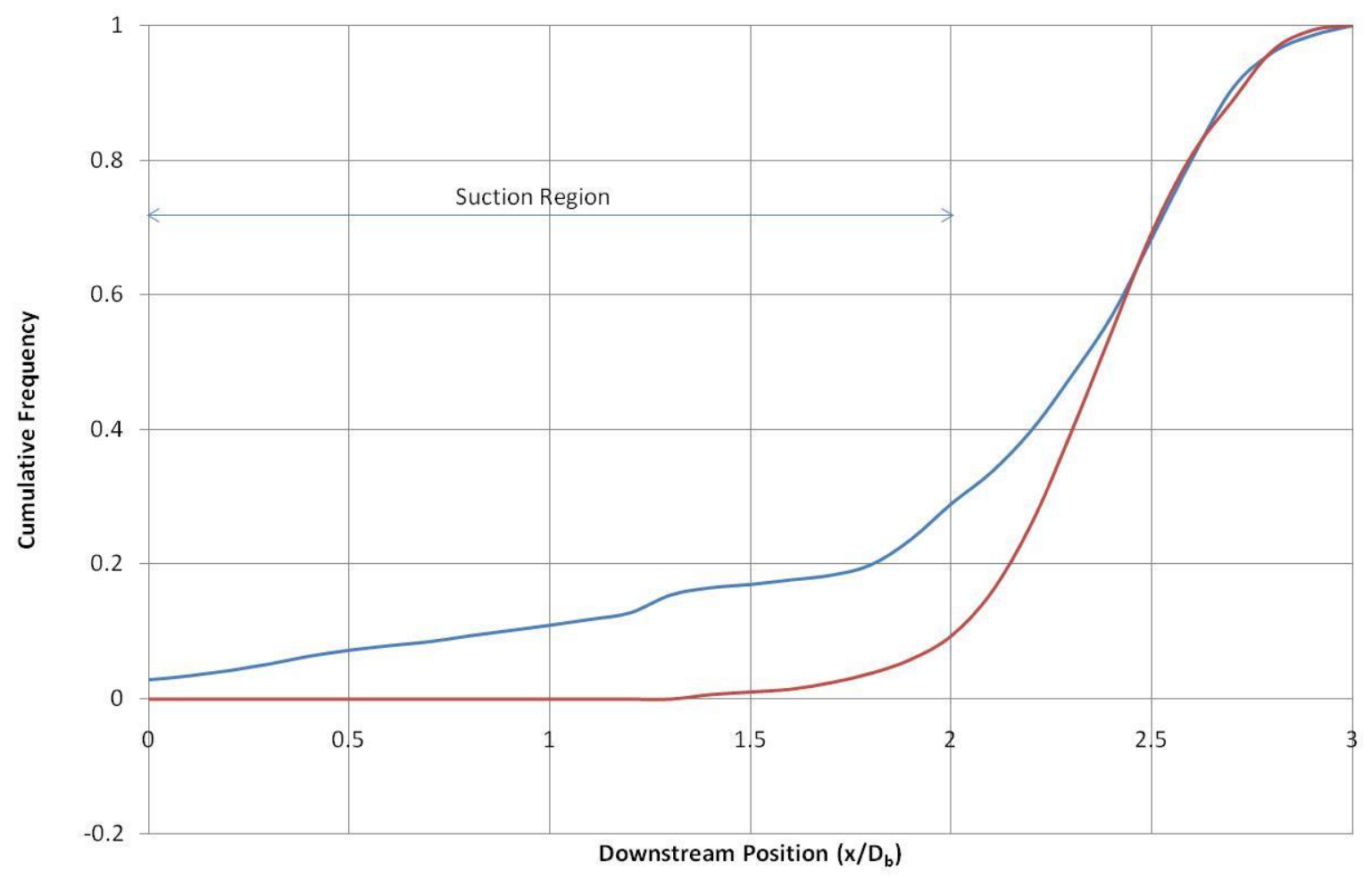

Figure 14. Cumulative frequency of nose position of flexible body, for two three minute experiments.

\subsection{Case 6 - Rigid Body, tension only tether, $3 D_{b}$ downstream, $U_{0}=0.42 \mathrm{~m} / \mathrm{s}$}

The observations of Liao et al. ${ }^{9}$ suggest that a flexible body is required to achieve Kármán gait like motion. Experiments with a rigid body manufactured from a wooden broom handle with the same dimensions as used in the previous experiments dispute this hypothesis. Figure 15 shows a 4.67 second sample where the rigid body 'swims' upstream in a similar motion to the Kármán gait. For the majority of the five minute run the tether spends very little time in tension. However, observations suggest that a flexible body with natural frequencies close to the shedding frequency are better able to interact with the shed vortices and thus spend a higher percentage of their time in a Kármán gait type motion. 

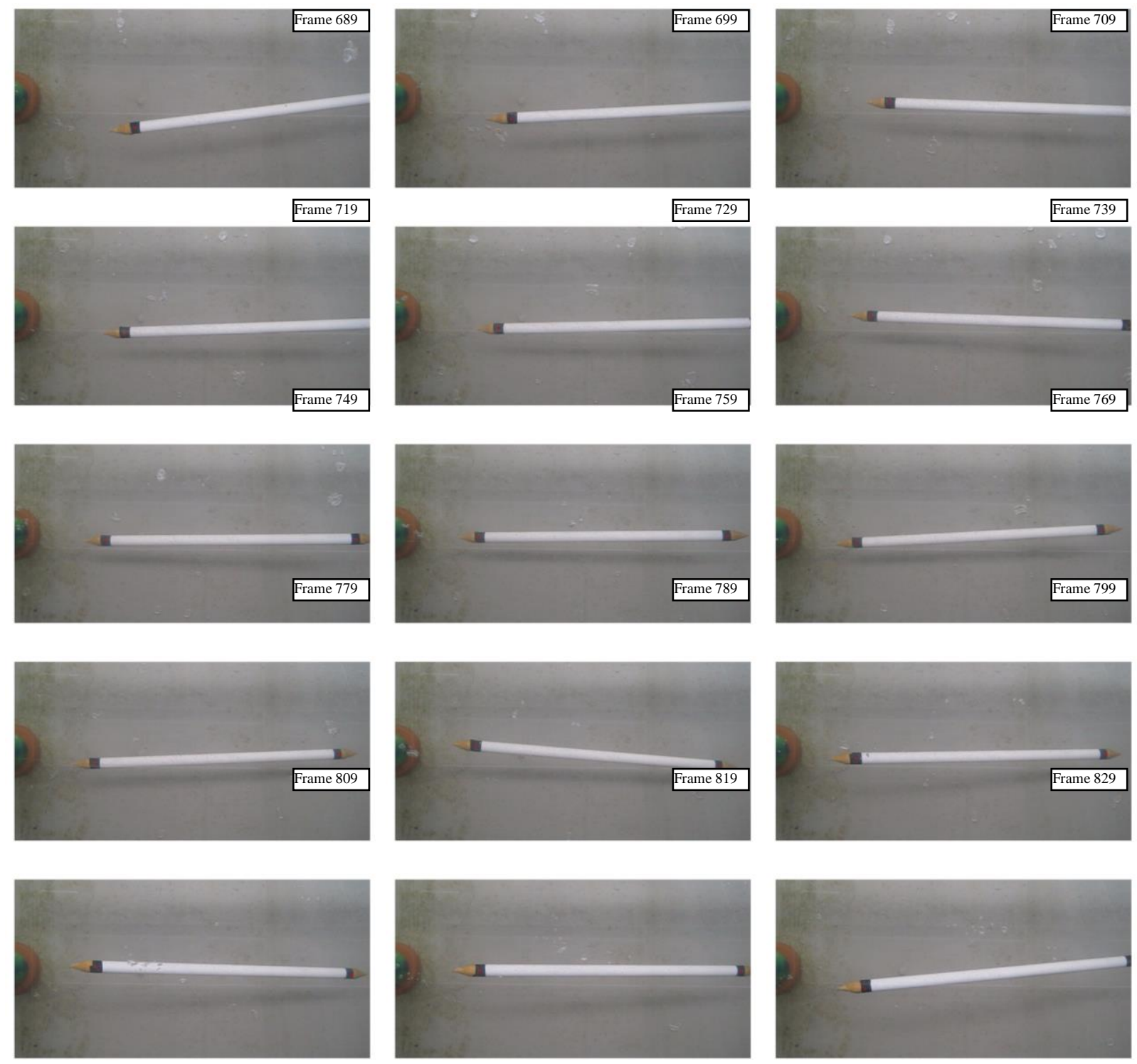

Figure 15. Case 6 - Rigid body, tension only tether, $3 D_{b}$ downstream, $U_{0}=0.42 \mathrm{~m} / \mathrm{s}$. Frames 689 to 829 separated by 10 frames $(0.3333 \mathrm{~s})$ intervals. The flow is passing from left to right.

As observed for the flexible body, the time histories of upstream location for the rigid body are highly dependent on whether the rigid body is drawn into the suction zone behind the cylinder. If caught in the suction region the body can spend significant periods of time with the nose very close or touching the rigid cylinder replicating the 'entrainment' behavior of fish. The ability of a rigid body to adopt a motion similar to the Kármán gait is potentially dependent on the length of the body in relation to the downstream vortex spacing. 


\section{Discussion}

The experiments have shown that both flexible and rigid bodies are able to passively interact with the Kármán wake in manners similar to those observed in fish, adopting behaviours similar to the Kármán gait and entrainment. If an AUV was able to adopt such behaviours in practise this would potential reduce the energy expenditure of the vehicle when operating in unsteady flows.

The power consumption of an AUV is highly dependent on the vehicle size, sensor package and operating speed. Typically it is assumed that the power consumption of an AUV can be split into the hotel load which is invariant to forward speed and the propulsion power which is proportional to velocity cubed ${ }^{15}$. For an AUV operating at its optimum speed associated with maximum range the propulsion power equates for approximately $1 / 3$ rd of the total power requirements. At higher speeds the propulsion power begins to dominate due to the associated velocity cubed term. For current small flight style AUVs the optimum speed is typically $1 \mathrm{~m} / \mathrm{s}$. It is therefore entirely feasible for a vehicle station keeping in a highly unsteady tidal stream or river adopting these behaviours may significantly reduce the propulsion power requirement and consequently make a significant impact on the vehicle total power consumption. However, in all these experiments the flexible body has been tethered to prevent the flexible body travelling too far downstream. All the time histories show that the tether becomes taut on various occasions for each of the time histories. As such it is unlikely that a truly passive device would be able to maintain station for extended periods of time. To achieve this, some additional actuation and an improved understanding of the surrounding fluid flow from additional sensors such as an artificial lateral line ${ }^{16,17}$ are required. This will add to the hotel load of the AUV and add an additional energy requirement to drive the actuators.

As behaviours for an operational AUV, both the Kármán gait and entrainment modes have advantages and disadvantages. Conceptually, any method of reducing the propulsive energy expenditure of an AUV will have a positive implication for the range of the vehicle as long as there is no associated increase in the vehicle's hotel load (propulsion speed-invariant power requirements) or displacement. Entrainment appears to be the easier behaviour to replicate but has the disadvantage of the nose of the vehicle being very close to or bumping against the rigid structure, which may lead to damage of the hull or exposed sensors (cameras, or conductivity temperature and depth sensors etc.). The Kármán gait would be a much more difficult behaviour to replicate 
with an operational AUV, but would have the advantage of maintaining a safe distance between the AUV and the rigid structure.

\section{Conclusions}

Simple motion capture and flow visualisation techniques have been applied to observe the motion of a flexible body tethered downstream of a rigid cylinder in the unsteady Kármán wake. Fourier transforms of the lateral motion of the flexible body show frequencies corresponding to the vortex shedding frequency of the rigid cylinder as well as the zeroth and first natural frequencies of the body. When tethered with a tension only tether, upstream motion was observed for both the flexible and cylinder rigid body in a mode which appears to replicate the Kármán gait observed in rainbow trout. However, a flexible body appears to allow greater exploitation of the Kármán gait. In many instances the interaction with the shed vortices and resulting upstream motion leads to the body being captured in the suction region behind the rigid cylinder where the body adopts entraining as a station-holding mechanism.

For AUVs operating in unsteady flow regimes, an ability to utilise energy saving gaits may improve the range or operational time of the vehicle. By either matching the drag on the body with thrust generated by the passing vortices, or by utilising the suction zones behind bluff bodies, upstream motion is achievable with no additional power expenditure. The results presented in this paper demonstrate the feasibility of such an approach for manufactured objects; however, further work is required to implement such behaviours on an operational vehicle, a key component of which will be providing the AUV with sufficient information about the local flow field to allow the control system to interact with the unsteady flow features.

\section{Acknowledgements}

This research was partially supported by the EPSRC through grant number EP/F066767/1 entitled "Nature in Engineering for Monitoring the Oceans (NEMO)" a joint project between the University of Southampton, Newcastle University and the National Oceanography Centre, Southampton. Aspects of this work were submitted by Jonathan Schembri in partial fulfilment of his Master of Science degree in Maritime Engineering Sciences in the School of Engineering Sciences at the University of Southampton. 


\section{References}

1. Webb PW. Entrainment by river chub Nocomis micropogon and smallmouth bass Microterus dolomieu on cylinders. Journal of Experimental Biology. 1998; 201: 2403-12.

2. Liao JC, Beal DN, Lauder GV and Triantafyllou MS. The Kármán gait: novel body kinematics of rainbow trout swimming in a vortex street. Journal of Experimental Biology. 2003; 2006: 1059-73.

3. Hinch SG and Rand PS. Optimal swimming speeds and forward-assisted propulsion: energyconserving behaviours of upriver-migrating adult salmon. Canadian Journal of Fisheries and Aquatic Sciences. 2000; 57: 2470-8.

4. Fausch KD. Experimental analysis of microhabitat selection by juvenile steelhead (Oncorhynchus mykiss) and coho salmon (O. kisutch) in a British Columbia stream. Canadian Journal of Fisheries and Aquatic Sciences 1993; 50: 1198-207.

5. McLaughlin RL and Noakes DLG. Going against the flow: an examination of the propulsive movements made by young brook trout in streams. Canadian Journal of Fisheries and Aquatic Sciences. 1998; 55: 853-60.

6. Lauritzen DV, Hertel FS, Jordan LK and Gordon MS. Salmon jumping: behavior, kinematics and optimal conditions, with possible implications for fish passageway design Bioinspiration \& Biomimetics. 2010; 5: 1-11.

7. Williamson CHK. Vortex Dynamics in the Cylinder Wake. Annual Review of Fluid Mechanics. 1996; 28: 477-539.

8. Liao JC, Beal DN, Lauder GV and Triantafyllou MS. Fish Exploiting Vortices Decrease Muscle Activity. Science. 2003; 302: 1566-9.

9. Liao JC. A review of fish swimming mechanics and behaviour in altered flows. Philosophical Transactions of the Royal Society B. 2007; 362: 1973-93.

10. Beal DN, Hover FS, Triantafyllou MS, Liao JC and Lauder GV. Passive propulsion in vortex wakes. Journal of Fluid Mechanics. 2006; 549: 385-402.

11. Phillips AB, Blake JIR, Smith B, Boyd SW and Griffiths G. Nature in engineering for monitoring the oceans: towards a bio-inspired flexible autonomous underwater vehicle operating in an unsteady flow. Journal of Engineering for the Maritime Environment. 2010; 224 267-78.

12. Toming G, Chambers LD and Kruusmaa M. Experimental study of hydrodynamic forces acting on artificial fish in a von Kármán vortex street. Underwater Technology: International Journal of the Society for Underwater. 2014; 32: 81-91.

13. Khalak A and Williamson CHK. Dynamics of a hydroelastic cylinder with very low mass and damping. Journal of Fluids and Structures. 1996; 10: 455-72.

14. Lighthill MJ. Hydromechanics of aquatic animal propulsion. Annual Review of Fluid Mechanics. 1969; 1: 413-46.

15. Phillips $\mathrm{AB}$, Haroutunian M, Man SK, et al. Nature in Engineering for Monitoring the Oceans: Comparison of the energetic costs of marine animals and AUVs. In: Roberts GN and Sutton R, (eds.). Further Advances in Unmanned Marine Vehicles. IET, 2012.

16. Yingchen Y, Nam N, Nannan C, et al. Artificial lateral line with biomimetic neuromasts to emulate fish sensing. Bioinspiration \& Biomimetics. 2010; 5: 016001.

17. Roberto V, Otar A, Francesco V, et al. Hydrodynamic pressure sensing with an artificial lateral line in steady and unsteady flows. Bioinspiration \& Biomimetics. 2012; 7: 036004. 